\title{
Neutron Scattering Studies of the Ferroelectric Transition in P (VDF-TrFE) Copolymers
}

\author{
Authors: J.F. Legrand, B. Frick, B. Meurer, V.H. \\ Schmidt, M. Bee, \& J. Lajzerowicz
}

This is an Accepted Manuscript of an article published in Ferroelectrics in September 1990, available online: http://www.tandfonline.com/10.1080/00150199008211433.

Legrand, J. F., B. Frick, B. Meurer, V. H. Schmidt, M. Bee, and J. Lajzerowicz. "Neutron Scattering Studies of the Ferroelectric Transition in P (VDF-TrFE) Copolymers." Ferroelectrics 109, no. 1 (September 1990): 321-326. doi: 10.1080/00150199008211433.

Made available through Montana State University's ScholarWorks scholarworks. montana.edu 
NEUTRON SCATTERING STUDIES OF THE FERROELECTRIC TRANSITION IN P(VDF-TrFE) COPOLYMERS.

J.F. LEGRAND and B. FRICK

Institut Laue-Langevin, 156x, 38042 Grenoble Cedex, France

B. MEURER

Institut C. Sadron, ONRS, 6 rue Boussingault,

67083 Strasbourg Cedex, France.

V.H. SCHMIDT

Department of Physics, Montana State University,

Bozeman, MT 59717, USA.

M. BEE and J. LAJZEROWICZ

Laboratoire de Spectrométrie Physique, Université J. Fourier Grenoble, B.P. 87, 38402 Saint Martin d'Hêres Cedex, France.

\section{INTRODUCTION}

In $P$ (VDF-TrFE) random copolymers with compositions from $60 / 40$ to $80 / 20$ mol 8 , the ferroelectric phase transition exhibits a strong first order character and is accompanied by a large expansion of the crystal lattice. In the ferroelectric structure, the polymer chains have an all-trans conformation and are close-packed parallel to one another in a pseudo-hexagonal lattice. According to the $m 2 m$ symmetry, the spontaneous polarization $P_{Y}$ is perpendicular to the chain axis $Z$. In the paraelectric phase, the structure is disordered : the chains undergo continuous conformational changes, they have a statistical $C_{\infty}$ symmetry and the expanded lattice is hexagonal $(6 / \mathrm{mmm}$ point group) ${ }^{1-3}$.

In this disordered high temperature phase, the molecular motions of large amplitude have been investigated using two different techniques :

(i) Nuclear magnetic resonance which probes the dynamics of reorientation of the chain segments ${ }^{4-7}$.

(ii) Incoherent quasi-elastic neutron scattering which probes the displacements of the individual protons (associated with the conformational changes of the chains $)^{8,9}$. 
The effect of hydrostatic pressure on the ferroelectric phase transition has also been investigated using neutron diffraction and NMR techniques.

\section{EXPERIMENTAL}

Copolymers with $70 \%$ VDF content were chosen for these studies, because they have a high degree of crystallinity (>70 8 ) and because the paraelectric phase extends over a large temperature range (from the melting point at $148^{\circ} \mathrm{C}$ down to the curie temperature at ${ }^{\circ} 65 \mathrm{C}$ ).

We used raw material (protonated) provided by Atochem Company (France) and by Solvay (Belgium) and also copolymers with selective deuteration on the VDF monomers which were synthetized by J.M. Kometani and R.E. Cais (AT and T Bell Laboratories). Unoriented powder specimens were investigated as well as polymer films oriented by rolling 5,10 .

The quasi-elastic neutron scattering experiments were performed on the backscattering Doppler spectrometer IN10, and the diffraction experiments under hydrostatic pressure on the high flux neutron diffractometer D20 (both instruments at the ILL, Grenoble, France).

\section{RESULTS AT ATMOSPHERIC PRESSURE}

From $19_{F}$ and $1_{H}$ NMR measurements of the spin-lattice relaxation times $T_{1}$ and $T_{1} \rho$ at different frequencies, the one-dimensional diffusion of the conformational changes (along the chain axis) was clearly evidenced, and it was shown that the dymanics of this diffusive motion in the paraelectric crystalline phase is very comparable to that in the intercrystalline amorphous phase ${ }^{6}$. The correlation time $\tau_{D}$ of the diffusive motion is estimated to be $\tau_{D}=15$ ps at $393 \mathrm{~K}$ and $70 \mathrm{ps}$ at $353 \mathrm{~K}$ (from Hunt and Powles correlation function).

From the incoherent neutron scattering results, the elastic part of the scattered intensity provides information on the geometry of the motions of the protons : Figure 1 shows the measured EISF at different temperatures for two orientations of a drawn copolymer film. The results are analyzed with a model of diffusion of the protons inside a restricted volume of cylindrical shape whose axis is parallel to the (001) chain axis. When the crystalline phase is still ferroelectric ( $345 \mathrm{~K}$ is just below the Curie temperature), less than 35 \& of the protons exhibit large amplitude motions (mainly the protons in the amorphous regions). In the paraelectric phase $(387 \mathrm{~K})$, most of the 


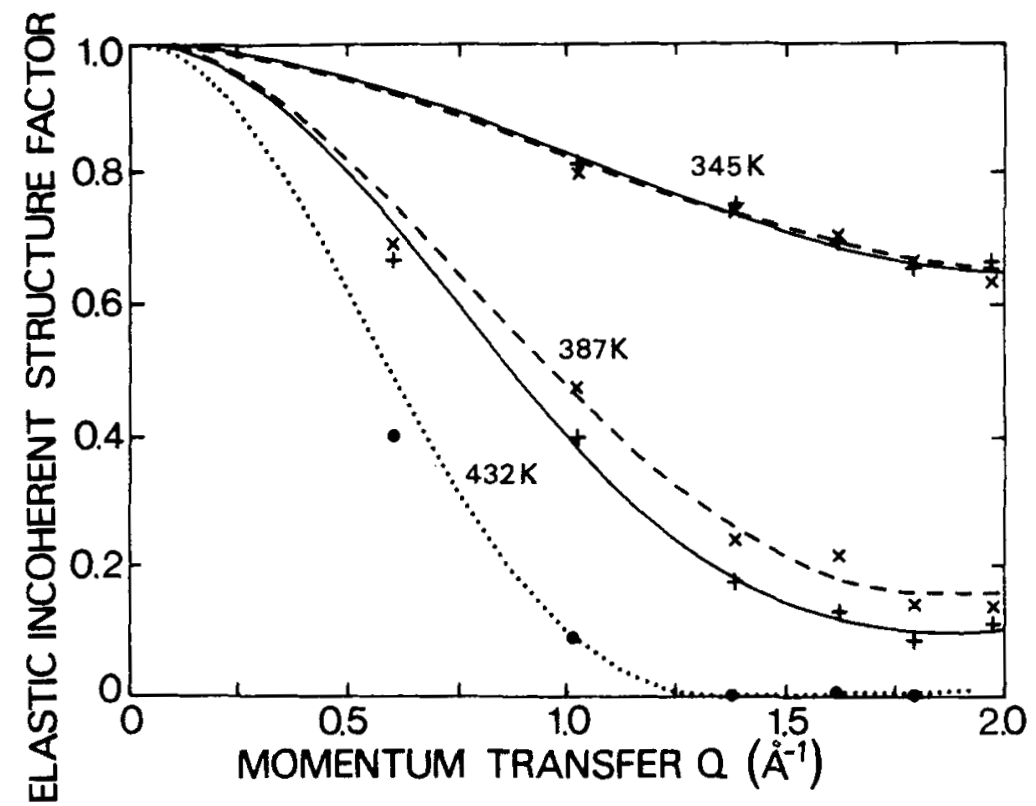

FIGURE 1 Experimental EISF for two orientations of a drawn specimen, and results of a fit with a model of difusion inside a cylindrical volume of radius $R$ and height $H$ :

$(+)$ and continuous lines correspond to the chain axis perpendicular to the scattering plane

(x) and broken lines correspond to the chain axis in the scattering plane.

Results provides by the fit:

- Ferroelectric phase at $345 \mathrm{k}$, $\mathrm{R}=1.5 \AA, \mathrm{H}=2.5 \AA$, $63 \%$ of fixed protons

- Paraelectric phase at $387 \mathrm{~K}, \quad R=2.0 \AA, H=3.3 \AA$,

- Molten phase at $432 \mathrm{~K}, \quad 0 \%$ of fixed protons.

protons display large amplitude motions with a component along the chain axis of comparable magnitude $(H=3.3 \AA$ ) as the component around the chain axis $(2 R=4 \AA)$. Above the melting point, in principle an EISF cannot be extracted, but the results obtained with the same treatment are shown to evidence the further increase in the amplitude of the motion. The energy width of the quasielastic part of the scattering gives an estimate of the average correlation time of the diffuse motion inside the restricted volume : $\tau_{\mathrm{c}}=200$ ps at $403 \mathrm{~K}$ and $\tau_{\mathrm{C}}=350 \mathrm{ps}$ at $353 \mathrm{~K}$. These are characteristic times for the protons to explore their average restricted volume, and the comparison with NMR results suggests that this motion consists of several jumps of amplitude of the order of $R\left(\tau_{D} / \tau_{C}\right)^{1 / 2}$, i.e. typically $0.8 \AA$ to $1.2 \AA$. 
With the selectively deuterated copolymer, the incoherent neutron scattering from the remaining protons (on TrFE monomers) shows essentially the same behaviour as observed in fully protonated samples and this confirms the cooperative nature of the one-dimensional excitations along the chains. Furthermore, deuteron NMR experiments were performed with the same specimen in the paraelectic phase ${ }^{7}$. The results support the model of kink-3-bond hindered reorientations propagating back and forth along chains having sequences of TG and $\overline{T G}$ conformations.

\section{RESULTS UNDER HYDROSTATIC PRESSURE}

The investigation of powder diffractograms versus temperature and pressure was recently performed using neutrons of $2.40 \AA$ wavelength. Over the whole observed range, the strong first order character of the ferroelectric transition remains with broad regions of phase coexistence clearly visible from the $(110)+(200)$ Bragg reflections. For the construction of the phase diagram shown in Figure 2, we represented the middle points of the coexistence regions. It is striking that the melting temperature and the curie temperature (upon heating) exhibit the same increase under pressure. This means that the enthalpy change (and the volume change) are very comparable for these two transitions, and this again points out the highly disordered nature of the paraelectric phase.

In this experiment, we could also analyze the changes of the lattice parameters versus pressure and temperature (Figure 3 ) and derive the lattice compressibility $x$ and its thermal expansion coefficient $\alpha$. In the ferroelectric phase we obtain $X=1.210^{-10} \mathrm{~Pa}^{-1}$ and $\alpha=1.310^{-4} \mathrm{~K}^{-1}$, while in the disordered paraelectric phase we obtain $X=2.510^{-10} \mathrm{~Pa}^{-1}$ and $\alpha=6.210^{-4} \mathrm{~K}^{-1}$ (these are volumic coefficients but one must stress that almost no change of the lattice parameter $\mathrm{c}$ is observed : it is the direction of the covalent -C-C-Cbondings).

Preliminary NMR results show that the spin-lattice relaxation time $T_{1}$ also decreases under hydrostatic pressure in the paraelectric phase, but with a $(P / T)$ scaling law which differs from that of the volume change. 

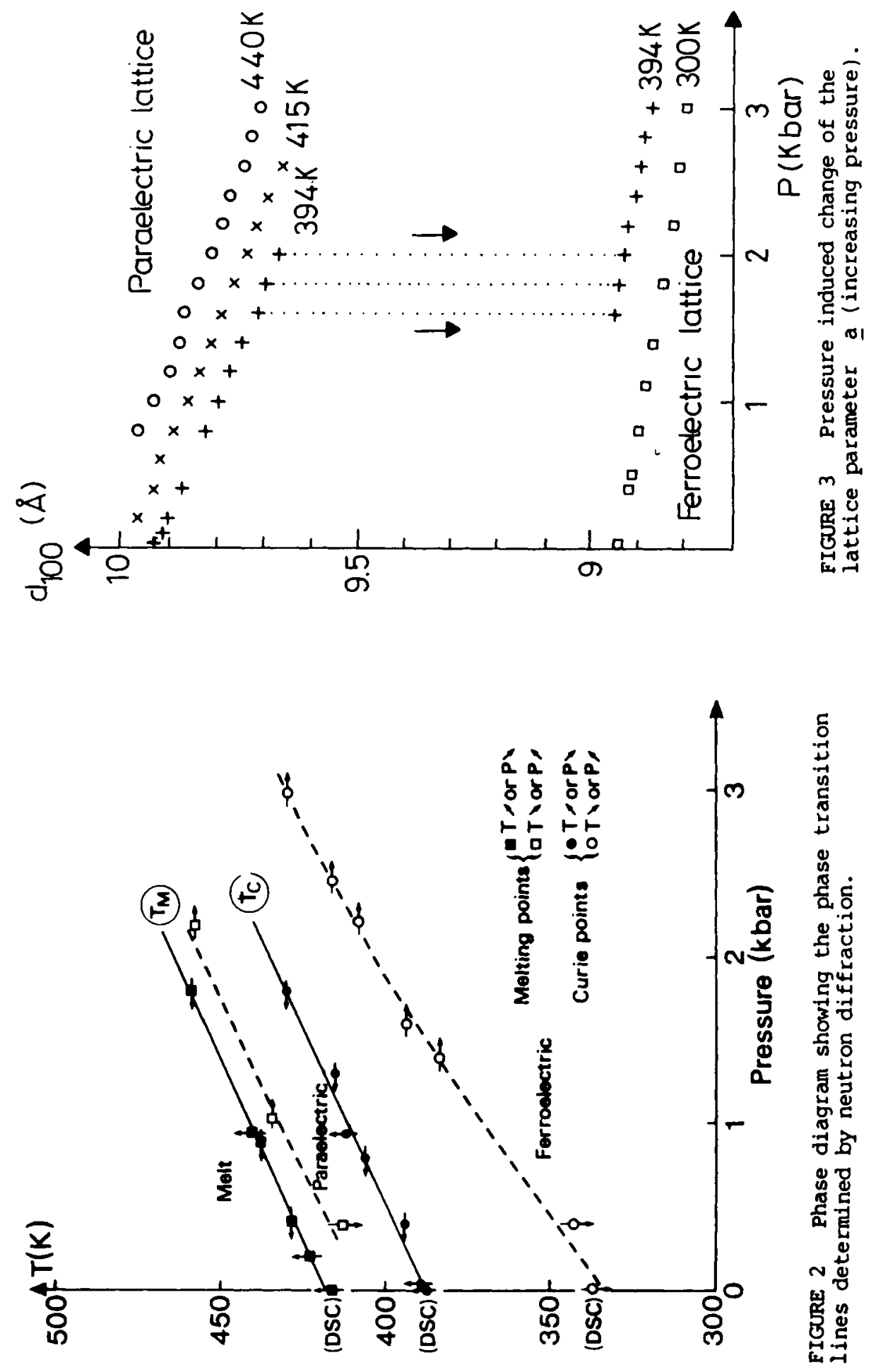


\section{CONCLUSION}

We have shown in this paper that neutron and MMR techniques provide complementary information on the molecular dynamics in the conformationally disordered phase of $\mathrm{P}$ (VDF-TrFE) copolymers. The main characteristics of the conformational changes have been obtained but further analyses would need molecular dynamics calculations and computer simulations. In this purpose, the results obtained under pressure are able to help in the analysis of the model outputs.

\section{REFERENCES}

1. T. Furukawa, G.E. Johnson, H.E. Bair, Y. Tajitsu, A. Chiba and E. Fukada, Ferroelectrics 32, 61 (1981).

2. A.J. Lovinger, T. Furukawa, G.T. Davis and M.G. Broadhurst, Polymer, 24, $1225 \& 1233$ (1983).

3. J.F. Legrand, J. Lajzerowicz, B. Berge, P. Delzenne, F. Macchi, C. Leonard, A. Wicker and J.K. Kruger, Ferroelectrics, 78, 151 (1988).

4. J.F. Legrand, P.J. Schuele, V.H. Schmidt and M. Minier, Polymer, $26,1683(1985)$.

5. J. Hirshinger, B. Meurer and G. Weill, Polymer, 28, 721 (1987).

6. J. Hirshinger, B. Meurer and G. Weill, J. Physique, 50, $563 \& 583$ (1989).

7. C. Perry, E.A. Dratz, Y. Ke, V.H. Schmidt, J.M. Kometani and R.E. Cais, Ferroelectrics, 92, 55 (1989).

8. J.F. Legrand, P. Delzenne, A.J. Dianoux, M. Bee, C. Poinsignon, D. Broussoux and V.H. Schmidt, Springer Proceedings in Physics, 29,59 (1988).

9. E. Lopez-Cabarcos, A. Gonzales-Arche, F. Baltallan and B. Frick, Physica B, 156, 423 (1989).

10. J.K. Kruger, J. Petzelt and J.F. Legrand, Colloid and Polymer Sci. 264,791 (1986). 